\title{
Research on Computer-aided Diagnosis Method of Obstructive Sleep Apnea Hypopnea Syndrome
}

\author{
Lingli Zhu * \\ College of Information Technology \\ Luoyang Normal University \\ Henan Luoyang, China \\ zhulinglils@163.com \\ * Corresponding Author
}

\author{
Huijuan Sun \\ Dept. of Information Engineering \\ Henan College of Finance \& Taxation \\ Henan Zhengzhou, China \\ huijuansunedu@163.com
}

\begin{abstract}
A computer-aided diagnosis system used for Obstructive Sleep Apnea Hypopnea Syndrome is designed. The technical realization of the system in the aspects of image segmentation, 3D reconstruction, data measurement and the like is mainly discussed. The system operability and effectiveness are analyzed through diagnosis study on 11 children cases of OSAHS. Experimental results show that the system can analyze the characteristics in the aspect upper airway morphology and function of OSAHS children, is beneficial for assisting the doctors in accurately locating the location, extent and degree of upper airway narrowing collapse of children patients. Its operability and effectiveness are preliminarily validated in the study.
\end{abstract}

$\begin{array}{cccc}\text { Keywords- } & \text { Obstructive } & \text { Sleep } & \text { Apnea Hypopnea } \\ \text { Syndrome;Image } & \text { Segmentation; } & 3 D \quad \text { Reconstruction; }\end{array}$ Computer-aided

\section{INTRODUCTION}

Obstructive Syndrome Sleep Apnea Hypopnea (OSAHS) is known as obstructive sleep apnea and hypoxia, which is caused by a lack of breathing and oxygen. OSAHS long-term repeated attacks can lead to hypoxia, high levels of blood, and the body's metabolism of adverse effects. There are many factors that cause OSAHS, which is the main reason for the collapse of the upper respiratory tract and local soft tissue. Generally speaking, the degree of stenosis of the pharyngeal cavity is proportional to the severity of OSAHS. The more weight of OSAHS, the smaller the minimum diameter of the pharyngeal cavity, and it is the smaller the minimum cross-sectional area. Operation treatment of OSAHS is the most effective treatment, so it is the key to improve the success rate of the upper airway stenosis or obstruction.

With the continuous development of medical imaging technology, CT scan is more and more widely used in clinical diagnosis and treatment because of its high resolution, and it can reflect the three-dimensional shape of human throat. It has become an effective method for detecting OSAHS [1-2]. But in clinical diagnosis, the doctor is often through one or more of the fault images to make a diagnosis, and did not make full use of the threedimensional information provided by CT images. The accuracy of this two dimensional detection system is largely determined by the clinical experience of doctors. In particular, judgment of children OSAHS patients with airway stenosis position, many researches mostly in the nasopharyngeal cavity parts, much to the size of the adenoid volume as the research direction by traditional methods such as Fujioka[3] and Zou Lai Ming Shun [4] to lateral nasopharyngeal X-ray by adenoid / nasopharynx ratio (A / $\mathrm{N}$ ratio) reaction adenoid body size, and describe the volume of nasopharyngeal airway, that the $\mathrm{A} / \mathrm{N}$ ratio can be as an indicator of the indication of adenoid hypertrophy and taking the opportunity as a criterion for evaluation of nasal pharyngeal airway stenosis. However, Powell et al. [5] has proved that in the adult, the flow shear stress of the tissue at the most narrow place is greatly increased.

This process may have a progressive damage to the receptors and other receptors in the upper airway and some of the receptors to coordinate the contraction of the diaphragm and respiratory muscles. Therefore, we believe that the traditional simply by measuring adenoid volume to evaluate children with OSAHS severity of surgical indications and the method has some defects. In addition, Major et al. [6] in the study indicated that the upper airway is a complex three-dimensional structure, and in children with OSAHS, because of the impact of disease, the upper airway morphology has a certain degree of variation, when the conversion to a two-dimensional image map will lose some information.

The OSAHS aided detection is performed by computer and the 3D model can be more comprehensive and visually reflect the stenosis of the children by the image segmentation and 3D reconstruction of CT. In addition, accurate upper airway morphology and functional status data is the premise of selecting reasonable treatment for patients. On the basis of the accurate positioning of the upper airway stenosis in patients with OSAHS is a necessary condition for surgical treatment. So it is very important to study the method of computer aided diagnosis of OSAHS. 


\section{DYNAMIC ADAPTIVE REGION GROWING ALGORITHM}

In order to realize the computer aided medical diagnosis and operation plan, the $3 \mathrm{D}$ reconstruction of human body image is needed. The primary task of 3D reconstruction is to extract the organs or tissues of interest. At present, there are many literatures on CT or MRI images. [7] uses the improved region growing algorithm to segment the organs of interest. [8,9] is used to segment liver CT images. Therefore, in view of the characteristics of the upper airway structure of OSAHS patients, the characteristics of the edge of the system, the system uses three-dimensional dynamic adaptive region growing algorithm, to achieve the rapid and accurate extraction of organs or tissues.

The basic idea of region growing is to set up a region of pixels with similar properties. A seed point is selected as the starting point of growth in the target region to be segmented, and then, in the field of seed points, the similarity degree of the seed points is searched, and the location of the seed points is merged. At this point, the newly merged pixels as the new seed point continue to search and merge the process, until no merger of the pixels so far. The key to this method is the location of the seed points, the growth rule and the growth order. At present, the research mainly focuses on the design of the characteristics of the measurement and the growth criterion, and to improve the efficiency and accuracy of the algorithm.

In this paper, a dynamic adaptive region growing algorithm is proposed. Firstly, a seed point (ROI) is selected as the initial point, and then the gray value of the $3 * 3$ neighborhood is calculated as the initial value of the seed region.

$$
\operatorname{mean}(x, y)=\frac{1}{9} \sum_{r=-1}^{1} \sum_{c=-1}^{1} g(x+r, y+c)
$$

At the same time, the variance of the neighborhood is calculated as the threshold of the growth criterion. The calculation formula is as follows:

$$
\delta=\sqrt{\sum_{r=-2}^{2} \sum_{c=-2}^{2}(g(x+r, y+c)-\operatorname{mean}(x, y))^{2}}
$$

Growth criteria

$|g(x, y)-\operatorname{avgGray}|<\delta$

The gray value of the pixel points to be incorporated in the formula (1), the center point coordinates of the seed region in the formula (2), and the average gray value of the current region in the type (3), the initial value is.

Let $\mathrm{s}$ be a seed point, $\mathrm{n}(\mathrm{x})$ said body $\mathrm{Su} x$ points in the neighborhood of the set, which said airway segmentation results, such as the initial value is null), STK is a stack, specific algorithms are as follows:

Stk.push(S);

Airway $=$ Airway $\cup S$;

While Stk is not empty

$$
\begin{aligned}
& \{\mathrm{x}=\operatorname{Stk} \cdot \operatorname{pop}() \\
& \text { For each } \mathrm{y} \in \mathrm{N}(\mathrm{x}) \\
& \{\text { if }|g(y)-\operatorname{avgGray}|<\delta \text { and } \mathrm{y} \notin \text { Airway } \\
& \quad\{\text { Stk. push }(\mathrm{y}) ; \\
& \quad \text { Airway }=\text { Airway } \cup \mathrm{y} ;\}\}\}
\end{aligned}
$$

Where $g$ (y) represents the gray level of the body y. Compared with the two-dimensional region growing method, the $\mathrm{N}(\mathrm{x})$ is replaced by a eight or four neighborhood of a 2D image to a six or twenty-six neighborhood of a 3D image.

This algorithm solves two key problems to be solved in the region growing algorithm:

(1) Selection of seed points

In the region growing algorithm, the user selects a single seed point, so the algorithm uses the gray mean value as the initial value of the seed region, which can avoid the error and noise. For the segmentation of adjacent slices, the method of seed region in place of single seed points is used.

(2) Determination of growth rules

Dynamic: with the continuous expansion of the growing region, the average gray value of the region is also followed by continuous change.

The adaptive threshold algorithm used in: growth is the growth variance region, adaptive.

\section{ROI AREA AND VOLUME CALCULATION}

According to the imaging principle of medical images such as CT and MRI, a pair of images can be viewed as a slice of a certain thickness of human tissue, which is represented by the thickness of the DICOM image. In this paper, the interval between the CT slices is represented by the layer spacing in the DICOM, and the values of these parameters are determined according to the setting of the imaging device. In medical image, a stereo ROI is composed of multiple planar ROI, which is present in multiple images. Thus the volume of the solid ROI can be expressed as the sum of the volume of the same name in the same name as the ROI slice of the same name. The volume of a planar ROI can be considered as a columnar body, with its area multiplied by the thickness of the layer, the area of each planar ROI is equal to the number of pixels in the ROI region of the DICOM region, which is expressed by a pixel on the body tissue of the body.

According to the results of the dynamic region growing, the number of pixels in ROI is calculated. By multiplying the number of pixels in the voxel space (i.e., the area of a pixel recorded in the DICOM file), the area of the upper airway plane can be calculated. In this paper, we calculate the cross section area of the upper airway CT slices, and the formula is as follows:

$S=$ Pixels $\times$ Voxel

Formula (4), the number of pixels within the ROI, the body space, that is, the image of each pixel representing the body area, this parameter can be obtained in the DICOM file. And the volume formula is:

$V=\sum_{i=1}^{n} S_{i} \times$ Thickness 
In this paper, the area of the first ROI area, the layer spacing between the sections, the layer spacing of CT slices in this paper is $0.625 \mathrm{~mm}$.

\section{THREE DIMENSIONAL RECONSTRUCTIONS}

Three dimensional reconstructions are the key to the realization of $3 \mathrm{D}$ visualization. The method of $3 \mathrm{D}$ reconstruction of medical image is mainly Direct Surface (Rendering) and Volume (Rendering). Surface rendering refers to the reconstruction of the surface of the object, which is extracted from the 3D data, and then uses the traditional graphics technology to fit the surface of the object. It can effectively draw three-dimensional body surface, and can draw the traditional computer graphics polygon rendering technology and hardware acceleration technology, so fast, but the lack of internal information expression. Direct volume renders, that is, the direct study of light through the body of the data field and the interaction between the body, without the need to construct the intermediate surface, and thus many of the details of the information to be retained, but time consuming.

This paper is aimed at the shortcomings of the existing code execution efficiency, low computational power, and the use of Visualization (Toolkit VTK), and the Cubes Marching method to extract the contour and 3D surface. In this method, the 3D object is decomposed into a cube, which is the same size as the body. After setting for the equivalent value, on the entire plain scan, judge the body element is contained in the isosurface is in the body element within the triangle approximation equivalent patch. Finally, each body element in small triangles connected constitutes objects of the isosurface shown on a twodimensional plane.

Three dimensional reconstructions are performed by $3 \mathrm{D}$ rendering. The reconstructed images are more vivid and more realistic, so it is easy to observe and analyze the internal organization, and save the 3D model of the upper airway as STL format. To the free edge of the soft palate, uvula, tip of the epiglottis free margin, vestibular splitting as a symbol, the key parts of the upper respiratory tract model is defined as: retro palatal region, uvula area, the lingual, epiglottis region, tracheal site.

\section{EXPERIMENTAL RESULTS AND DATA ANALYSIS}

In this paper, the patients from Guangzhou City, Guangdong province from January 2014 to January 2013, 11 patients with OSAHS disease, including 7 males and 4 females, aged 3-14 years old, average 7 years old. Data collection from the hospital department of radiology using PHILPS Brilliance 64 row CT scan data. Scan method: supine position, head advanced. Listen to the breath, nose line as the baseline, collected from the nasopharynx to supraglottic the volume data of the upper respiratory tract.

Image segmentation using the previously mentioned dynamic adaptive region growing method and it is the segmentation effect as shown in Figure 1 below. From the graph, the algorithm of this paper is very good for the segmentation of CT images, and the results are satisfactory. Compared with the traditional manual method, this method is more accurate, more real and faster, especially suitable for the auxiliary diagnosis.
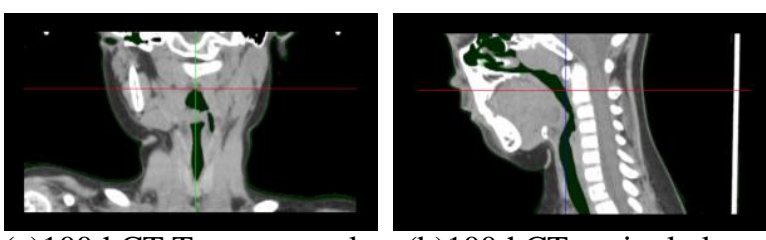

(a)100thCT Transverse plane(b)100thCT sagittal plane

Figure 1. Segmentation Effect of Dynamic Adaptive Region Growing.

D visualization results are shown in Figure 2, figure 3, figure 4 and figure 5 . The process uses a three-dimensional surface rendering method to reconstruct and render the surface of the upper airway. The model has good effect, and the shape is lifelike. It also can be displayed with the model combination of the face and other parts of the nose, and the transparency and color, which is helpful to the clinician to experience the anatomic structure of the upper airway and the spatial structure of each other. It is more suitable for the study of three-dimensional visualization (Figure 2 and figure 3). Three dimensional reconstructions will be more comprehensive and intuitive to reflect the upper airway stenosis, which has important clinical value. As a means of evaluating the degree of stenosis of the patient, it can be used to evaluate the extent of the stenosis of the patients (Figure 4). While the right of the patient and the upper airway and the same plane of the upper airway, it is more meaningful to define the extent of the stenosis. In addition, 3D reconstruction of 3D surface model generated by $3 \mathrm{D}$ reconstruction can be used as the basis for many studies of STL.

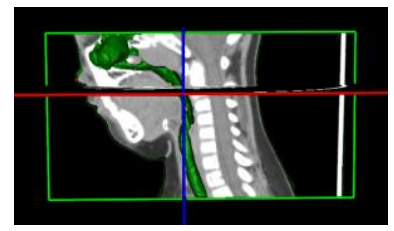

Figure 2. D model and 3D Interaction of CT

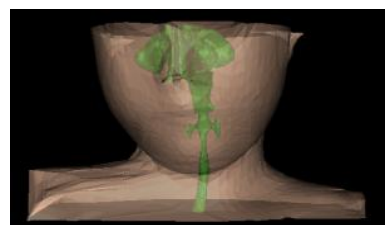

Figure 3. Transparent Display and Multi Angle Observation

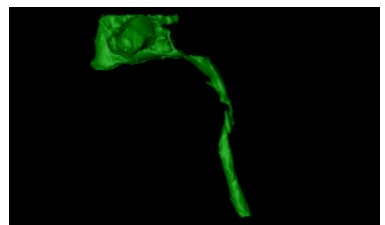

Figure 4. Three Dimensional Reconstruction Results of Awake State

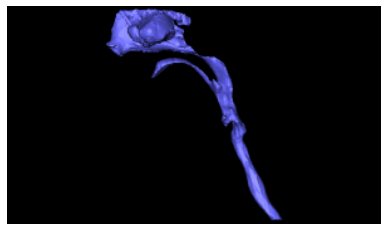

Figure 5. Three Dimensional Reconstruction Results of Sleep State

The semi automatic calculation of the smallest crosssectional area of the upper airway can accurately and 
quickly find the narrow position of the patient. The minimum cross-sectional area of the pharyngeal cavity was smaller than that of the normal people, but the OSAHS level of OSAHS was smaller. The stenosis degree of pharyngeal cavity was proportional to the severity of OSAHS. Using this three-dimensional visualization method, it can be used in the clinical application of threedimensional anatomical structure analysis of children's OSAHS before surgery, operation value, risk prediction, etc., compared with other relevant research methods have obvious advantages.

TABLE I. THE VOLUME OF AIRWAY AND THE SMALLEST CROSSSECTIONAL AREA OF THE UPPER AIRWAY AND THE AIRWAY OF OSAHS CHILDREN.

\begin{tabular}{ccccc}
\hline Case & \multicolumn{2}{c}{$\begin{array}{c}\text { Upper airway volume } \\
\left(\mathrm{mm}^{3}\right)\end{array}$} & \multicolumn{2}{c}{$\begin{array}{c}\text { Airway minimum cross- } \\
\text { sectional area }\left(\mathrm{mm}^{2}\right)\end{array}$} \\
\hline & Awake & Sleep & Awake & Sleep \\
1 & 18.00 & 15.00 & 43.00 & 22.00 \\
2 & 25.00 & 20.00 & 57.00 & 24.00 \\
3 & 25.00 & 17.00 & 59.00 & 27.00 \\
4 & 13.00 & 9.00 & 63.00 & 45.00 \\
5 & 13.00 & 12.00 & 66.00 & 29.00 \\
6 & 25.00 & 23.00 & 63.00 & 29.00 \\
7 & 21.00 & 12.00 & 74.00 & 46.00 \\
8 & 31.00 & 19.00 & 132.00 & 48.00 \\
9 & 15.00 & 10.00 & 118.00 & 102.00 \\
10 & 21.00 & 19.00 & 66.00 & 44.00 \\
11 & 27.00 & 16.00 & 155.00 & 75.00 \\
\hline
\end{tabular}

The data of CT in 11 cases were collected, and the image segmentation, 3D model reconstruction, area volume automatic calculation, and so on. The results showed that the upper airway volume and the crosssectional area of the upper airway volume and the crosssectional area of the airway were significantly less than that of the 11 patients with $\mathrm{T}$, and the difference was statistically significant $(\mathrm{t}=5.257, \mathrm{P}<0.01 ; \mathrm{t}=3.999, \mathrm{P}<$ 0.01). Traditional Fujioka method using a / $\mathrm{N}$ ratio to measure, namely in the basilar clivus skull outside do line of all, take the adenoid the convex point $\mathrm{B}$ and tangent to do a vertical point of intersection for a measured distance $A B$ is a value, $A B$ line extension of the nasopharyngeal airway lumen of palatal junction point $C$, distance of $A C$ is $\mathrm{n}$ value.

This paper on 11 cases by traditional Fujioka method [8], the average a / $\mathrm{N}$ value of 0.71 , including 6 cases of children who have a / $\mathrm{N}$ is more than or equal to 0.7 , accounting for only $54.5 \%$ of the total number of children. So we believe that the traditional $\mathrm{A} / \mathrm{N}$ ratio may not accurately judge the degree of stenosis of children's upper airway, the reason we analyze that, from the perspective of three-dimensional shape, the shape of the upper airway in children with OSAHS, the shape of the upper airway is more than the size of a wedge, although the diameter of the gland is the most narrow, but the right and left sides of the wall is not necessarily narrow, so its size is not the smallest.

The results of the experimental study can be revealed: the compliance of the pharyngeal wall of OSAHS patients is increased, and the possibility of collapse of the pharyngeal cavity is more likely. This change in a certain extent can reflect the differences of the upper airway compliance of the children with a clear and sleep state, and objectively reflect the location, extent and degree of stenosis. Preoperative diagnosis system of patients with upper respiratory tract stenosis or obstruction of the site to make accurate judgments, and then develop a more targeted surgical scheme is the key to improve the success rate of surgery.

Especially for children with a narrow, narrow, only to solve a problem and can not really improve the symptoms of children. Only accurate and comprehensive localization of the upper airway stenosis in children with a variety of treatment methods, and it is to clear the surgical indications, to correctly predict the effect of treatment. From this point of view, it has a unique advantage in the diagnosis of the upper airway of children with OSAHS.

\section{SUMMARY}

In this paper, the CT image segmentation and 3D modeling, the location, range, degree and direction of the upper airway stenosis were observed. Secondly, the calculation of the sectional area of the upper airway can be found accurately by using the correlation formula. The calculation of the cross-sectional area can accurately find out the upper airway stenosis plane, and the volume of the upper airway can reflect the change of airway volume. This is helpful to the study of the characteristics of the upper airway morphology and the accuracy of the clinical diagnosis of OSAHS. In addition, the operability and effectiveness of the system was preliminarily verified in the present study. The experimental data of 11 cases of OSAHS children showed that the three-dimensional computer aided diagnosis is a good method for the study on the morphology and function of the upper airway.

\section{ACKNOWLEDGMENT}

This paper is supported by Science and technology project of Henan Province (152102210123, 142102210475).

\section{REFERENCES}

[1] Chen NH, Li KK, Li SY, etal. Airway assessment by volumetric computed tomography in snorers and subjects with obstructive sleep apnea in a far-East Asian population (Chinese) [J].Laryngoscope, 2002, 112(4):721-726.

[2] TIAN Li, LIN Di. The association of adenoid and tonsil with obstructive sleep apnea hypopnea syndrome in children[J]. ACTA UNIVERSITATIS MEDICINALIS NANJING ( Natural Science) , 2010, 30(1):98-101

[3] Fujioka M, Young LW, Gird any BR.Radiographic evaluation of adenoidal size in children: adenoidal-nasopharyngeal ratio[J].American journal of roentgenology, 1979, 133(1):401-404.

[4] Zou Ming-shun. The adenoid-nasopharynx ratio:its clinical value in children[J]. CHINESE JOURNAL OF RADIOLOGY, 1997, 31(3):190-192.

[5] Major MP, Flores MC, Major PW.Assessment of lateral cephalometric diagnosis of adenoid hypertrophy and posterior upper airway obstruction: a systematic review[J].Am J Orthod Dentofacial Orthop, 2006, 130(6):700-708.

[6] Xu C, Sin S, McDonough JM. Computational fluid dynamics modeling of the upper airway of children with obstructive sleep apnea syndrome in steady flow[J].Journal of biomechanics, 2006, 39(1):2043-2050.

[7] YU Meng, LIU Xiang-rong, HUANG Xiao-yang, CHEN Yingchao. An Improved Region Growing Algorithm and Its Application 
in Coronary Artery Angiographic[J].Journal of Xiamen University(Natural Science), 2013,52(1): 38-42

[8] SONG Hong, WANG Yong. A Dynamic Adaptive Region Growing Segmentation Algorithm for Tumor of Liver CT Images[J]. Transactions of Beijing Institute of Technology, 2014,34(1):72-76
[9] LI Zhen-wei, LIU Bing-quan, ZHU Jian-min. Design and Realization of Contour Extraction of Liver CT Images Based on Genetic Algorithm[J]. Computing Technology and Automation, 2005,24(4):102-104 